\title{
REVERSIBLE INTEGER KLT FOR PROGRESSIVE-TO-LOSSLESS COMPRESSION OF MULTIPLE COMPONENT IMAGES*
}

\author{
Pengwei Hao ${ }^{1,2}$ \\ Qingyun $\mathrm{Shi}^{1}$ \\ ${ }^{1}$ Center for Information Science, Peking University, Beijing, 100871, China \\ ${ }^{2}$ Department of Computer Science, Queen Mary, University of London, London, E1 4NS, UK \\ e-mail: phao@cis.pku.edu.cn, phao@dcs.qmul.ac.uk
}

\begin{abstract}
In this paper, we presented a method for integer reversible implementation of KLT for multiple component image compression. The progressive-to-lossless compression algorithm employed the JPEG-2000 transform coding strategy using the multiple component transform (MCT) across the components, followed by a 2-dimensional wavelet transform on individual eigen images. The linear MCTs we tested and compared are KLT, discrete wavelet transform (DWT), and a tasselled cap transform (TCT) for TM satellite images only. The computational complexity of the reversible integer implementation is no more than that of naïve transformation, and the overhead data is very small. Its effectiveness was evaluated using two 6-band Landsat TM satellite images and an 80component hyper-spectral remotely-sensed image. Experiments with KLT and wavelet based JPEG-2000 show that reversible KLT (RKLT) outperforms other approaches for all of the test images in the case of both lossy and lossless compression.
\end{abstract}

\section{INTRODUCTION}

A multiple component image is a data set with two spatial dimensions and one component or spectral dimension. Multispectral images and hyper-spectral images are examples. Multiple component images usually possess a large amount of inter-band correlation due to natural spectral correlation, topographic slope and aspect, and overlap of spectral sensitivities between adjacent spectral bands.

Principal components analysis transform or KarhunenLoeve transform (KLT) are utilized as a tool to extract the significant inter-band information and eliminate the redundancy. KLT has been widely applied in many applications [8], such as image enhancement, feature

\footnotetext{
* This work was supported by NKBRSF China under Grant G1998030606 and the funding for the recipients of the National Excellent Doctoral Dissertation, China, under Grant 200038.
}

extraction, image classification, image segmentation, historical change detection, content-based retrieval, principal component analysis and image compression. KLT is a linear transformation which uses the signal statistics to define a rotation of the original images in such a way that the new axes are orthogonal to each other and point in the direction of decreasing order of the variances, and the rotated covariance matrix is diagonalized. The transformed components are totally uncorrelated.

Multiple component transformation (MCT) is one key step in multiple component image coding. KLT has been employed as an MCT in multispectral image coding for long. Typically, a compression technique applies KLT in the spectral dimension followed by spatial transform. Experiments in [5] show that compression with a spectral KLT followed by spatial DWT (discrete wavelet transform) outperforms that with spectral KLT and spatial DCT (discrete cosine transform) [7]. Reversible integer implementation of an MCT is the reasonable way for progressive lossy-to-lossless compression of multiple component images. However, even the recent paper [1] still takes KLT as a lossy transform.

A reversible integer transform is necessary in transform-based lossless compression, progressive lossyto-lossless transmission, unified lossy/lossless image coding system, and lossy compression with the lossless reproduction of a region of interest [3]. In light of these applications, we can see that there is a clear need to consider some reversible integer implementation for known linear transforms which are good enough for data decorrelation. It is well-known that a wavelet transform can be factorized into a series of lifting steps which can be implemented reversibly with integers [4]. We have mathematically proved that any linear transform can be implemented by reversible integer mapping [6], and the necessary and sufficient condition for a matrix to be factorized into a series of elementary reversible matrices (ERMs) is that the absolute value of the matrix determinant is 1 . And we also found some efficient ways for the factorizations, such as triangular ERM (TERM) and single-row ERM (SERM) factorizations.

In this paper, we presented an approach to factorize a KLT matrix into SERMs, and applied the method to 
multiple component transforms, followed by a spatial compression with wavelet-based JPEG-2000 [3]. Its effectiveness for both lossy and lossless compression was evaluated using two 6-band Landsat TM images and an 80-component hyper-spectral image. The linear transforms we tested and compared are KLT, DWT, and a tasselled cap transform (TCT) for TM (Thematic Mapper) satellite images only.

\section{MULTIPLE COMPONENT TRANSFORMS}

A lot of linear transforms can be defined and applied for transformation and inter-band decorrelation across the components of multiple component images, such as a discrete wavelet transform (DWT), Karhunen-Loeve transform (KLT).

The wavelet filter employed as the MCT in our experiments is Cohen-Daubechies-Feauveau bi-orthogonal $5 / 3$ filter, which can be implemented integer-reversibly by lifting scheme for both lossy and lossless compression.

KLT is a transform depending upon the data to be transformed. Its matrix consists of the eigenvectors derived from of the covariance matrix of all the data, so a KLT matrix is an orthogonal matrix, whose determinant is \pm 1 and the inverse is its transpose. From a viewpoint of least mean square error by discarding the high index coefficients in the transformed space and least energy in the smallest number of the coefficients, KLT is the optimal statistical transform and the most effective technique for data decorrelation. KLT produces the principal components of an image. The highest energy is concentrated in the transformed bands corresponding to the largest eigenvalues of the covariance matrix.

6-band Thematic Mapper (TM) images are acquired by satellite or aircraft mounted sensors. For TM remotely sensed images, there is another transform found by Crist and Cicone to extract the ground object information of luminance, green and moisture from infrared reflectance images, which is called tasselled cap transform (TCT). The whole matrix is made up with other orthogonal vectors:

$\boldsymbol{T}=\left[\begin{array}{cccccc}0.3037 & 0.2793 & 0.4743 & 0.5585 & 0.5082 & 0.1863 \\ -0.2848 & -0.2435 & -0.5436 & 0.7243 & 0.0840 & -0.1800 \\ 0.1509 & 0.1973 & 0.3279 & 0.3406 & -0.7112 & -0.4572 \\ -0.8242 & 0.0849 & 0.4392 & -0.0580 & 0.2012 & -0.2768 \\ -0.3280 & 0.0549 & 0.1075 & 0.1855 & -0.4357 & 0.8085 \\ 0.1084 & -0.9022 & 0.4120 & 0.0573 & -0.0251 & 0.0238\end{array}\right]$

It is also an orthogonal matrix, and we have $\operatorname{det} \boldsymbol{T} \approx 1$.

Of course, we can also define more real or complex transforms and more simple transforms, such as GramSchmidt transform matrix, and other linear prediction transforms. However, KLT is optimal for what concerns spectral decorrelation and information compaction.

\section{REVERSIBLE INTEGER MAPPING}

For a transform that maps an integer number $x$ to an integer number $y, y=a x+b$, the necessary and sufficient condition for reversible mapping is $|a|=1$, and $\mathrm{b}$ is an integer number. We name a number of $|a|=1$ as an integer factor, which is denoted as $j$, and its reciprocal $j^{\prime}=1 / j$. For real number mapping, the condition is $a= \pm 1$ and using a rounding arithmetic for $b,[b]$, where [ ] is an arithmetic rounding operation (rounding or chopping or some bits before decimal point). The flow chart of the transform and its inverse is illustrated as in Fig. 1.

In the case of $a=1$, or $j=1$, the structure is the same as the ladder structure proposed by Bruekers \& van den Enden [2] and the lifting scheme structure by Daubechies \& Sweldens [4].

In [6], we have proved that any $N$-by- $N$ square matrix $\boldsymbol{A}$ whose determinant is \pm 1 can be factorized into a product of $N+1$ SERMs. A unit SERM can be formulated as $\boldsymbol{S}_{m}=\boldsymbol{I}+\boldsymbol{e}_{m} \boldsymbol{s}_{m}^{T}$, where $\boldsymbol{e}_{m}$ is the $m$-th standard basis vector formed as the $m$-th column of the identity matrix, $\boldsymbol{s}_{m}=\left\{s_{m n}\right\}(n=1,2, \cdots, N)$ is a vector that the $m$-th element $s_{m m}$ is 0 . The unit SERMs share an important property: $\boldsymbol{S}_{m}^{-1}=\boldsymbol{I}-\boldsymbol{e}_{m} \boldsymbol{s}_{m}^{T}$. For expression convenience, we denote $\boldsymbol{S}_{0}=\boldsymbol{I}+\boldsymbol{e}_{N} \boldsymbol{s}_{0}^{T}$ in our following discussion. So the SERM factorization can be $\boldsymbol{A}=\boldsymbol{P} \boldsymbol{S}_{N} \boldsymbol{S}_{N-1} \cdots \boldsymbol{S}_{1} \boldsymbol{S}_{0} \boldsymbol{J}$, where $\boldsymbol{P}$ is a permutation matrix for partial pivoting, $\boldsymbol{J}$ is a diagonal matrix whose diagonal elements are all integer factors.

If complete pivoting is applied, the SERM factorization can also be $\boldsymbol{A}=\boldsymbol{P}_{L} \boldsymbol{S}_{N} \boldsymbol{S}_{N-1} \cdots \boldsymbol{S}_{1} \boldsymbol{S}_{0} \boldsymbol{P}_{R} \boldsymbol{J}$.

The implementation with $N+1$ SERMs has a flowchart structure as Fig. 2 illustrated.

Since we have

$$
\begin{aligned}
\boldsymbol{A} & =\boldsymbol{P}_{L} \boldsymbol{S}_{N} \boldsymbol{S}_{N-1} \cdots \boldsymbol{S}_{1} \boldsymbol{S}_{0} \boldsymbol{P}_{R} \boldsymbol{J} \\
& =\left(\boldsymbol{P}_{L} \boldsymbol{P}_{R}\right)\left(\boldsymbol{P}_{R}^{T} \boldsymbol{S}_{N} \boldsymbol{P}_{R}\right)\left(\boldsymbol{P}_{R}^{T} \boldsymbol{S}_{N-1} \boldsymbol{P}_{R}\right) \cdots\left(\boldsymbol{P}_{R}^{T} \boldsymbol{S}_{1} \boldsymbol{P}_{R}\right)\left(\boldsymbol{P}_{R}^{T} \boldsymbol{S}_{0} \boldsymbol{P}_{R}\right) \boldsymbol{J} \\
& =\left(\boldsymbol{P}_{L} \boldsymbol{S}_{N} \boldsymbol{P}_{L}^{T}\right)\left(\boldsymbol{P}_{L} \boldsymbol{S}_{N-1} \boldsymbol{P}_{L}^{T}\right) \cdots\left(\boldsymbol{P}_{L} \boldsymbol{S}_{1} \boldsymbol{P}_{L}^{T}\right)\left(\boldsymbol{P}_{L} \boldsymbol{S}_{0} \boldsymbol{P}_{L}^{T}\right)\left(\boldsymbol{P}_{L} \boldsymbol{P}_{R}\right) \boldsymbol{J}
\end{aligned}
$$

we can also use other SERM factorization forms, such as

or

$$
\begin{gathered}
\boldsymbol{A}=\boldsymbol{P} \boldsymbol{S}_{k_{N}} \boldsymbol{S}_{k_{N-1}} \cdots \boldsymbol{S}_{k_{1}} \boldsymbol{S}_{k_{0}} \boldsymbol{J} \\
\boldsymbol{A}=\boldsymbol{S}_{k_{N}} \boldsymbol{S}_{k_{N-1}} \cdots \boldsymbol{S}_{k_{1}} \boldsymbol{S}_{k_{0}} \boldsymbol{P J}
\end{gathered}
$$

where $k_{0}=k_{N}$, and $k_{N}, k_{N-1}, \cdots, k_{2}, k_{1}$ is a permutation of $N, N-1, \cdots, 2,1$.

For each SERM, $\boldsymbol{S}_{m}$, its reversible integer transform can be implemented in-place for $m$-th element only:

$$
y_{m}=x_{m}+j_{m}\left[\sum_{n \neq m} s_{m n} x_{n}\right]=x_{m}+j_{m}[b]
$$

where $m=1,2,3, \cdots, N$.

The reversible version of KLT is referred to as RKLT in this paper. 
The advantages of the reversible integer implementation are: (1) Best approximation of given linear transform; (2) Integer reversible (information lossless); (3) In-place calculation (add to: $+=$ ); (4) Flexible rounding arithmetic $(\mathrm{x}+=[\mathrm{b}])$; (5) Simple inverse (subtract from: -=); (6) Acceptable computation complexity.

\section{COMPUTATIONAL COMPLEXITY AND OVERHEAD INFORMATION}

The integer reversible transforms are calculated using float operations, but rounding arithmetic and in-place integer assignments. Clearly, reversible implementation introduces no more additional FLOPS than its original direct linear transform. Moreover, every transform built with SERMs is immediately invertible, and its inverse has exactly the same computational complexity as the forward transform. For an $N$-by- $N$ linear transform matrix, the computational complexity of MCTs is given in Table 1 .

There is some overhead information associated with RKLT that must be recorded in the encoded bit stream. For an $N$-component image, to transform with our factorized matrices, the overhead is $N^{2}-1$ floating-point numbers for SERMs, $N$ integer numbers for permutation and $N$ one-bit tags for integer factors. If 4-byte floating numbers and one-byte integer numbers are used, we need $8\left(4\left(N^{2}-1\right)+N\right)+1=32 N^{2}+8 N-31$ bits per image. For a 6 -band $512 \times 512$ image, the uncompressed overhead data should be $(32 \times 6 \times 6+8 \times 6-31) /(512 \times 512 \times 6)=0.0007$ bits/pixel. It's not expensive.

\section{EVALUATION METHODOLOGY}

For evaluation purposes, the JPEG-2000 technique is used for compression of each transformed component. The test strategy is shown in Fig. 3.

The tested MCTs are DWT, reversible tasselled cap transform (RTCT), KLT, reversible KLT (RKLT). We also test the performance without any transform (NoT). The wavelet filter employed in the JPEG-2000 encoder is Cohen-Daubechies-Feauveau bi-orthogonal 5/3 filter for lossy and lossless compression.

The test images used in our experiments are listed in Table 2. Images of Beijing and Powerplant are two sub-images of a Landsat TM image, consist of 6 bands of the same resolution, namely band $1,2,3,4,5$ and 7 . Image $\mathrm{XB}$ is a high-resolution and hyperspectral image acquired by PHI(Pushbroom Hyperspectral Imager), radiometric corrected. It was acquired by Institute of Remote Sensing Applications, Chinese Academy of Sciences, China.

For the transforms under consideration, the final bit rate measured by bits per pixel per band (bpppb) is used to evaluate the performance of lossless compression. For lossy compression, the test images are encoded at several bit rates, 0.5, 0.25, 0.125, and $0.0625 \mathrm{bpppb}$, and an objective image distortion metric, peak signal-to-noise ratio (PSNR), is used for evaluation:

$$
P S N R=10 \log _{10} \frac{N \cdot 255^{2}}{\sum_{k=1}^{N} \operatorname{MSE}\left(B_{k}\right)}
$$

In order to display the contrast between the direct KLT and its integer reversible implementation, RKLT, we also losslessly compress the images transformed with the original lossy KLT and the residual images (eKLT), and then take the total bit rates (KLT+eKLT) as the lossless performance of lossy KLT.

\section{EXPERIMENTS}

Image quality test is undertaken as discussed in the previous section. The test results of three multiple component images are shown in Tables 3 and 4. The relatively best performance is highlighted with bold digits.

Each of the test images listed in Table 2 is compressed in a lossless manner using the MCTs, and the results are shown in Table 3.

Through the results we can see that RKLT has the best performance in the case of lossless compression. The fixed transform RTCT is the second best for TM images.

All the test images listed in Table 2 are also compressed in a lossy manner at 4 fixed bit rates using the MCTs, and the results are shown in Table 4.

All the results share one conclusion: RKLT is the best for lossless compression overwhelmingly and very close to KLT for lossy compression, and RTCT is a little worse than RKLT for TM images, but still excellent.

\section{CONCLUSIONS}

Our proposed integer reversible implementation method of multiple component transform outperforms the original linear transform calculations. It gives a possibility to apply a linear transform losslessly in image coding. In our experiments, the reversible KLT has acceptable computational complexity and gives the best performance for lossless compression and very close to non-reversible KLT for lossy compression. An image-independent fixed transform, TCT, is also excellent for TM images. Therefore, it is very possible to find one proper transform and its integer implementation for a very class of multiple component images.

\section{REFERENCES}

[1] A. Benazza-Benyahia; M. Hamdi, J.-C Pesquet, "Vectorlifting schemes for lossless coding and progressive archival of 
multispectral images", IEEE Trans. Geoscience and Remote Sensing, V40, N9, pp. $2011-2024$, Sep 2002

[2] F. A. M. L. Bruekers, A. W. M. van den Enden, "New networks for perfect inversion and perfect reconstruction", IEEE J. on Selected Areas in Comm., V10, N1, pp. 130-137, 1992.

[3] C. Christopoulos, A. Skodras, T. Ebrahimi, "The JPEG2000 still image coding system: An Overview”, IEEE Trans. Consumer Electronics, V46, N4, pp. 1103-1127, 2000

[4] I. Daubechies, W. Sweldens, "Factoring wavelet transforms into lifting steps", J. of Fourier Analysis and Application, V4, N3, pp 247-269, 1998.

[5] P. L. Dragotti, G. Poggi and A.R.P. Ragozini, "Compression of Multispectral Images by three-dimensional SPIHT Algorithm”, IEEE Trans. Geosci. Remote Sensing, V38, N1, pp. 416-428, January 2000.

[6] P. Hao and Q.Y. Shi, "Matrix Factorizations for Reversible Integer Mapping", IEEE Trans. Signal Processing, V49, N10, pp. 2314-2324, Oct. 2000.

[7] J. A. Saghri, A. G. Tescher, and J. T. Reagan, "Practical transform coding of multispectral imagery," IEEE Signal Processing Magazine, V12, N1, pp. 32-43, Jan. 1995.

[8] A. Singh, and L. Eklundh, "Comparative analysis of standardised and unstandardised principal components analysis in remote sensing", Int. J. on Remote Sensing, V14, pp. 13591370, 1993.

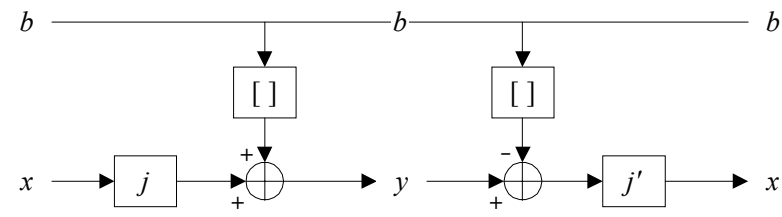

Fig. 1 Forward and reverse linear transform of a number implemented by integer mapping

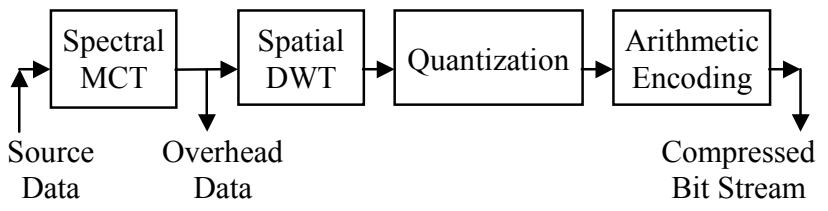

Table 1: Computational complexity comparison

\begin{tabular}{|c|c|c|c|c|}
\hline Transform & Adds & Multiplies & Rounds & Permutation \\
\hline DWT(5/3) & $15 N / 4$ & $15 N / 8$ & $15 N / 8$ & Unnecessary \\
\hline KLT & $N^{2}-N$ & $N^{2}$ & $N$ & No \\
\hline RKLT & $N^{2}-1$ & $N^{2}-1$ & $N+1$ & Needed \\
\hline
\end{tabular}

Table 2: Test Images

\begin{tabular}{|c|c|c|c|}
\hline Image Name & Size & Bands & Description \\
\hline Beijing & $512 \times 512$ & 6 & Urban areas of Beijing \\
\hline Powerplant & $512 \times 512$ & 6 & $\begin{array}{c}\text { Vegetation, mountains, } \\
\text { power plant with smoke }\end{array}$ \\
\hline XB & $346 \times 512$ & 80 & Road, water, rice paddy \\
\hline
\end{tabular}

Table 3: Lossless compression (Bit Rate, bpppb)

\begin{tabular}{|c|c|c|c|c|c|c|c|}
\hline Image & NoT & DWT & RTCT & RKLT & KLT+eKLT & KLT & eKLT \\
\hline Beijing & 4.29 & 4.04 & 3.93 & $\mathbf{3 . 7 7}$ & 5.31 & 3.70 & 1.61 \\
\hline Powerplant & 3.98 & 3.84 & 3.75 & $\mathbf{3 . 6 7}$ & 4.96 & 3.57 & 1.39 \\
\hline XB & 5.51 & 4.95 & -- & $\mathbf{4 . 8 9}$ & 6.38 & 4.84 & 1.54 \\
\hline
\end{tabular}

Table 4: Lossy compression (PSNR, dB)

\begin{tabular}{|c|c|c|c|c|c|c|}
\hline \multirow{2}{*}{$\begin{array}{c}\text { Bit Rate } \\
\text { (BPPPB) }\end{array}$} & \multirow{2}{*}{ Image } & \multicolumn{4}{|c|}{ Multiple Component Transform } \\
\cline { 2 - 7 } & & NoT & DWT & RTCT & RKLT & KLT \\
\hline \multirow{4}{*}{0625} & Beijing & 32.14 & 32.70 & 32.69 & $\mathbf{3 3 . 2 3}$ & 33.12 \\
\cline { 2 - 7 } & Powerplant & 32.74 & 33.18 & 33.32 & $\mathbf{3 3 . 6 4}$ & 33.45 \\
\cline { 2 - 7 } & XB & 19.27 & 25.03 & -- & $\mathbf{2 6 . 7 0}$ & 26.64 \\
\hline \multirow{4}{*}{0.125} & Beijing & 33.15 & 33.76 & 33.89 & $\mathbf{3 4 . 6 3}$ & 34.50 \\
\cline { 2 - 7 } & Powerplant & 34.03 & 34.52 & 34.74 & $\mathbf{3 5 . 0 7}$ & 34.82 \\
\cline { 2 - 7 } & XB & 21.38 & 26.75 & -- & $\mathbf{2 8 . 3 1}$ & 28.24 \\
\hline \multirow{4}{*}{0.25} & Beijing & 34.47 & 35.38 & 35.61 & $\mathbf{3 6 . 5 7}$ & 36.44 \\
\cline { 2 - 7 } & Powerplant & 35.67 & 36.34 & 36.67 & $\mathbf{3 7 . 1 2}$ & 36.80 \\
\cline { 2 - 7 } & XB & 23.96 & 28.96 & -- & $\mathbf{3 0 . 3 0}$ & 30.21 \\
\hline \multirow{4}{*}{0.5} & Beijing & 36.27 & 37.46 & 37.75 & $\mathbf{3 8 . 9 9}$ & 38.86 \\
\cline { 2 - 7 } & Powerplant & 37.73 & 38.54 & 39.01 & $\mathbf{3 9 . 6 8}$ & 39.16 \\
\cline { 2 - 7 } & XB & 27.27 & 31.68 & -- & $\mathbf{3 2 . 6 9}$ & 32.58 \\
\hline
\end{tabular}

Fig. 3 Encoding strategy

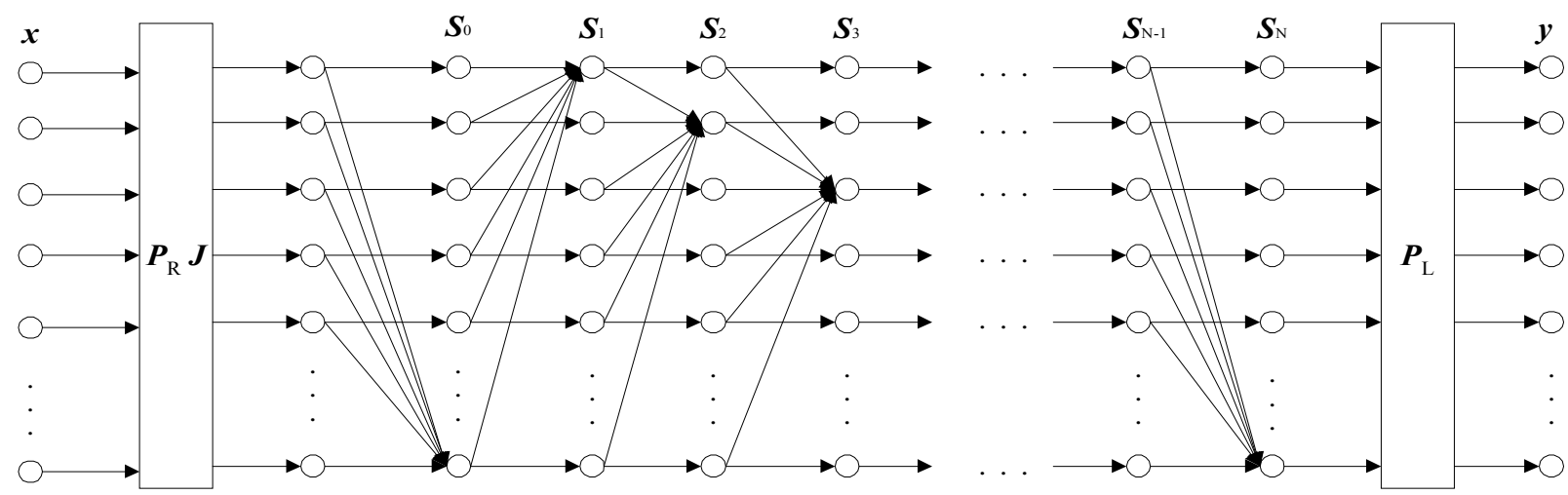

Fig. 2 A flowchart structure of linear transform implemented by SERMs 